\title{
A Multiscale and Anisotropic Edge Detection Algorithm
}

\author{
Hua-Jie Cai ${ }^{1}$ \\ China Ship Development and Design Center \\ Wuhan, China \\ e-mail: 349144467@qq.com
}

Tao $\mathrm{Li}^{2}$

School of Automation

Huazhong University of Science and Technology

Wuhan, China

e-mail: litao_2011@hust.edu.cn

\begin{abstract}
Edge detection is of important significance for computer vision. Current edge detection methods based on the first derivative or second derivative such as Canny and Laplace and Gaussian operators use single scale information and do not take account of the edge directions sufficiently. Those operators cannot distinguish edges and noise well. A multiscale and multidirectional edge detection algorithm is proposed for gray images in this paper. Gaussian function is used as the filter kernel. A serials scales and directional filters are generated by the generating function. A bank of edge maps are acquired by convoluting the original image with those filters. The maximum response is used to find the local maximum in the maps. Finally, the edges are determined after a certain threshold. The experiments show that the proposed algorithm gets outperformance compare to some state of art methods.
\end{abstract}

Keywords- Edge detection; multiscale filter; maximum response; Canny operator; scale multiplication .

\section{INTRODUCTION}

With the rapid development of computer vision, Image feature extraction becomes more important. Image edges carry the structure information of the whole scene, playing the key role in feature representation. Lots of researches about edge detection have been done [1]-[6]. The basic process of image detection is to evaluate the neighborhood of the pixel and then get the local maxima. The evaluation is usually carried out by a derivative based template. Some classical approaches used a fixed filter template, such as Sobel[5], LOG[6]. Others used a dynamic template size, such as Canny operator [1]. Generally, the dynamic size corresponds to scale of the edges in the image. The edge detection in the scale space often has a better result since edges and noise present different characteristics in scale space. Canny edge detection method is considered as one of the most successful edge detection method and is widely used in the engineering applications. Canny defined three criteria of edge detection: low error rate, good localization and uniqueness of the response. And based on the three criteria, he proposed an edge detection method by using first derivative of Gaussian (FDOG) and dual-threshold.

\author{
Xin $\operatorname{Tian}^{3}$ \\ School of Electronic Information \\ Wuhan University \\ Wuhan, China \\ e-mail: xin.tian@whu.edu.cn
}

The traditional Canny operator has some drawbacks. It has a poor effect on impulsive noise due to the Gaussian smoothing filter used before gradient operator. Hence, the impulsive noise is easy mistaken for edges and the signal to noise ratio become lower. Another defect is the dualthreshold set. A high threshold and low threshold are both used by Canny. Edge detection effect is very sensitive to the threshold setting. Some weak edges cannot be detected if the threshold is too high. However, if the threshold is too low, many of the non-edge pixels will be erroneously detected as edges. The thresholds in Canny operator are set manually which has a poor adaptability. It easily leads to missing or mistake.

Paul [7] enhanced Canny operator using a scale multiplication. A scale multiplication function is defined in his work, and it implied that scale multiplication had better results than a single scale used by Canny, especially for the localization criterion. Paul improved detection performance defined by the three criterions but it is still not a perfect work. Edges exist in different scale space. The whole contour information exists in large scales while the tiny edges for the image details exist in small scales. However, only two scales were used in Paul's work that cannot reflect the complete edge information. Furthermore, the local maxima are obtained by gradient operator as the same as Canny's work which just includes horizontal and vertical directions. Edges on other directions will have weaker local maximum response. Hence, it is difficult to distinguish them from the noise. In other words, the direction information of edges is not full used.

In this work, a multiscale and anisotropic edge detection algorithm is proposed. First, a bank of multiscale FDOG filters are generated which have different scale of $\mathrm{x}$-direction and y-direction, and then the multiscale FDOG filters are rotated to some directions. Several maps are generated by the convolution of those filters and an image. We get the maximum response of those directions in each scale. Finally, we get another bank of maps by scale multiplication of every two scales and we choose the best one of scale multiplication maps as the edge map. The proposed edge detection scheme is showed in figure 1. 


\section{The Proposed Edge Detect AlgorithM}

\section{A. Multiscale Techniques on Edge Detection}

Scale space is proposed by Lijima[8] in 1962 . The basic idea of scale space is to introduce the 'scale parameter' in image processing models and get different image information through a series of continuous scales. Compared with traditional single-scale processing technology, multiscale space methods work with the dynamic analysis framework, making it easier to get the essence of the image. The classical multiscale methods usually extract the main contours in serial scales, and then structure them into feature vectors. The corners and edges can be analyzed in the multiscale feature set[11],[12]. Gaussian scale space is proved to be the only liner scale space by Lingdeberg[9], which is presented by

$$
G(x, y)=A e^{-\frac{x^{2}+y^{2}}{2 \sigma^{2}}}
$$

where $\sigma$ is the scale parameter. Calculate the partial derivatives of $G(x, y)$ in x-direction and y-direction, respectively, in order to get edge detection convolution kernel, represented by

$$
\begin{gathered}
G_{x}=2 A x e^{-\frac{x^{2}+y^{2}}{2 \sigma^{2}},}, \\
G_{y}=2 A y e^{-\frac{x^{2}+y^{2}}{2 \sigma^{2}}} .
\end{gathered}
$$

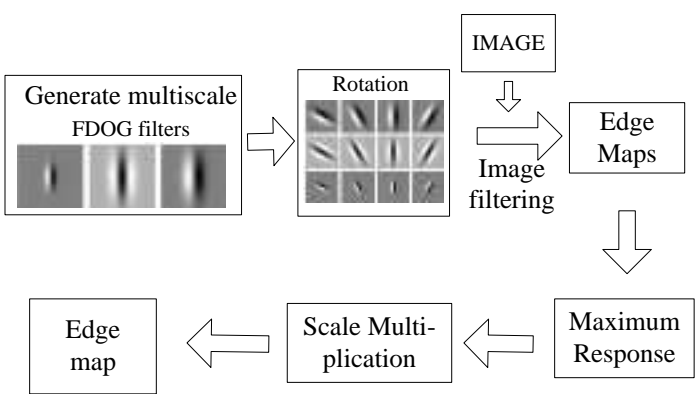

Figure 1. The scheme of the proposed algorithm

Serial edge maps are acquired after the two directional kernels convolute with image $I(x, y)$, which can be described as

$$
\begin{aligned}
& H(x, y)=G_{x} * I(x, y), \\
& V(x, y)=G_{y} * I(x, y) .
\end{aligned}
$$

Then, the intensities is defined by

$$
T(x, y)=\sqrt{H^{2}(x, y)+V^{2}(x, y)} .
$$

A series of edge maps can be obtained by using different scale parameter $\sigma$. Figure 2 show the derivatives of
Gaussian kernel in different scales and figure 3 show some edge map samples with several scales.

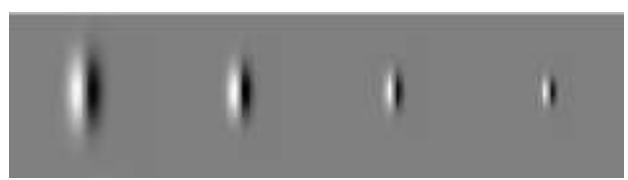

(a)

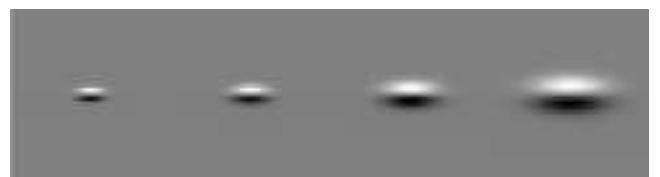

(b)

Figure 2. The horizontal and vertical derivatives of Gaussian. (a) The horizontal derivative of Gaussian with four scales. (b) The vertical derivative of Gaussian with four scales.

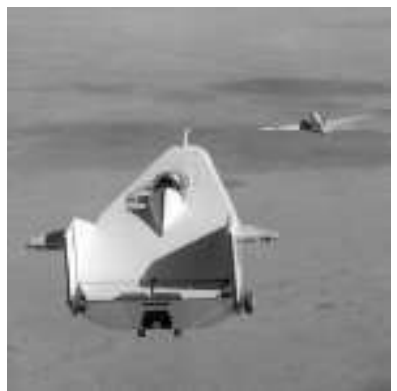

(a)

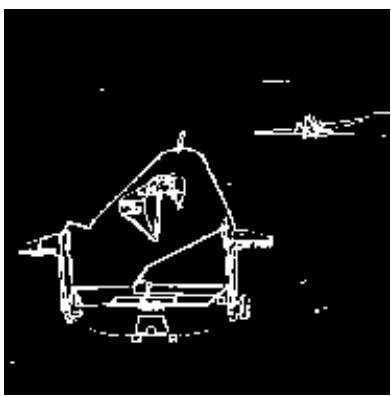

(c)

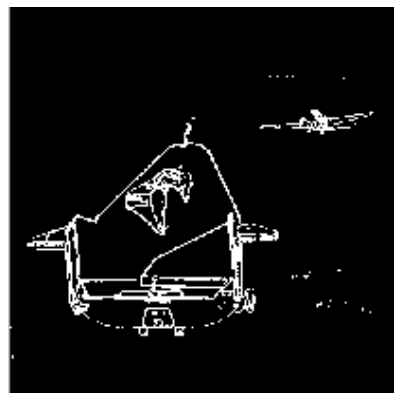

(b)

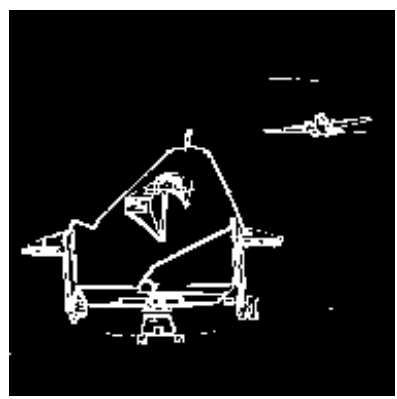

(d)
Figure 3. Image convolution with different scales. (a) Original image

(b) Edge map with the scale $\sigma=\sqrt{2} / 2$. (c) Edge map with the scale $\sigma=\sqrt{2}$. (d) Edge map with the scale $\sigma=2 \sqrt{2}$.

\section{B. Directional filtering and Scale multiplication}

As noted above, Gaussian filter is isotropic, and FDOG filters only have horizontal and vertical directions which are always replaced by the gradient operator. Edges have different directions ranging from 0 to $\pi$ while noise is usually not sensitive in directions. Hence, only the horizontal and vertical directions are not enough to distinguish edges and noise. For example, given two edge points have the same intensity values and adjacent relationship, one point is in vertical direction and the other is in $\pi / 3$ direction. They could have different response when do the convolution using $G_{x}$ or $G_{v}$. It is not conducive 
to edge detection. More directions are needed for all the edges. The proposed directional filter is part of the LM filter bank [10], which are the rotation of FDOG,

$$
G^{\prime}=G_{x} \cos \theta+G_{y} \sin \theta
$$

where $\theta$ is the angle between the major axis of the directional filter and $\mathrm{x}$-axis. The precondition for directional filters is that the scale $\sigma_{x}$ for $G_{x}$ is not the same as the scale $\sigma_{y}$ for $G_{y}$. Otherwise $G$ is isotropic. That is, there is no main scale. The direction is more obvious if the difference of $\sigma_{x}$ and $\sigma_{y}$ is bigger. Some directional filters in different scales are showed in figure 4.

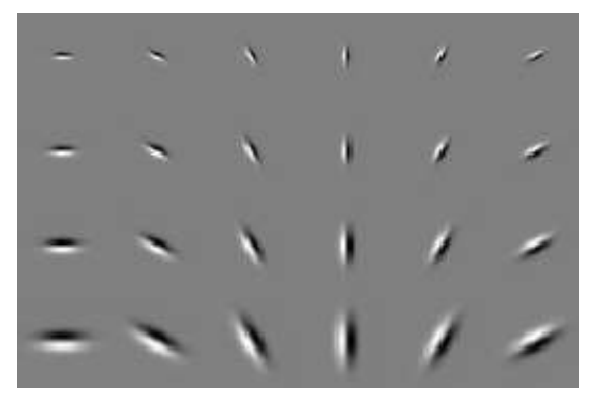

Figure 4. Directional FDOG with four scales and six directions.

Scale multiplication enhances the edge detection effect further as stated by Paul[7]. He used two scales denoted by $s_{1}$ and $s_{2}$ to convolute with the image $I(x, y)$ and denoted $H^{s_{1}}(x)$ and $H^{s_{2}}(x)$ as the responses of the convolution. Then the scale multiplication is defined as

$$
P(x)=H^{s_{1}}(x) \cdot H^{s_{2}}(x) .
$$

He stated that the scale multiplication could enhance edge and suppress noise so that his algorithm got more efficient and robust results. The simulation result of 1-dimentional signal is shown in figure 5.

Paul used the scale multiplication in canny operator directly. It may be not a fair treatment for all the directions. Instead, in the proposed method, we apply it in multiscales and multidirections.

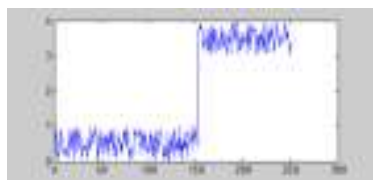

(a)

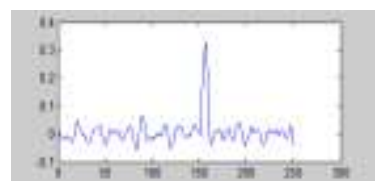

(c)

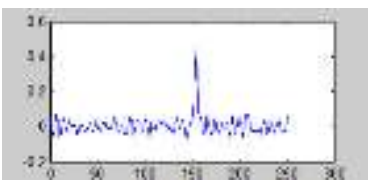

(b)

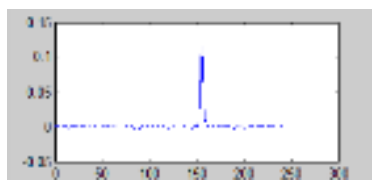

(d)
Figure 5. Scale multiplication on 1-Dimentional signal. (a) The original signal. (b) Signal filtered with scale $\sigma_{1}$. (c) Signal filtered with scale $\sigma_{2}$ (d) The result of scale multiplication

\section{RESULTS AND ANALYSIS}

Two experiments have done in this part. One is to detect the edges with low noise; the other is to test with heavy noisy image. Four scales and six directions are used to get enough edge details, which are $\theta=\{0, \pi / 6, \pi / 3, \pi / 2,2 \pi / 3,5 \pi / 6\} \quad$ and, $\sigma=\{\sqrt{2} / 2, \sqrt{2}, 2,2 \sqrt{2}\}$, totally 24 filters. Hence, 24 maps are acquired after the convolution. We get the maximum response of all the directions in each scale. Then, each scale has only one edge map. Two adjacent scales are multiplied to avoid loss of edge details. We also provide three compared experiments, which are Sobel, Canny, and literature [7].

Fig.6 shows the edge detection results on 'cameraman.bmp'. Sobel and Canny are executed by Matlab 7.0 tools, using the function 'edge'. The two scales in literature [7] are set 0.5 and 1 . It can be seen that all of those methods can achieve a satisfied detection result on 'cameraman.bmp'. Only minor differences present in details. Canny get continuous edges due to its edge connection skills. Other differences are mainly caused by the different thresholds set in those methods. It can be concluded that simple edge detection should be used when the edges in the image are clear and the noise is in low level. The execution complexity is the key factor considered for this kind of image.

The edge detection result always depends on the noise level in the image. The traditional methods such as Sobel are very sensitive to all kinds of noises. While Gaussianbased methods are more robust to noise due to the smoothing filtering. Fig .7 shows the noise impact on edge detection. The Gaussian noise and impulse noise are added in the synthetic image. We adjust the thresholds until they get the best visual effect for each method. As can be seen from Fig .7, Sobel gets the worst result which lots of noise are mistaken for edges since it doesn't get noise suppression. The result of Canny is acceptable for vision, but it still has a few noises in the edge map mainly caused by the impulse noise. Literature[7] and the proposed method both have a good performance and the proposed is better. Our algorithm achieves the best results.

\section{CONCLUSION}

This paper proposed a multiscale end directional filtering method to improve the edge detection effect. Compared to some single scale methods such as Canny or Sobel, multiscale of FDOG filters are used in order to get more edge information in the scale space. Edges have obvious directions while noise is not that regular. Hence noises can be well suppressed due to the directional filters. Furthermore, scale multiplication technique enhances our detection results. The local maxima can be obtained by maximum response method. Finally, a threshold is set to 
get the real edges. The experiments demonstrate we get better results in terms of noise suppression and edge location accuracy compared to some well-known algorithms such as Canny. Nevertheless, the proposed algorithm still needs improvement. The computational complexity is too high and the adaptability is not good which we will continue to study in our feature work.

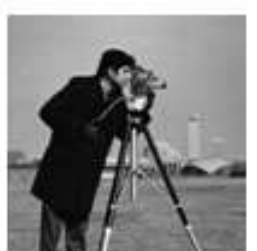

(a)

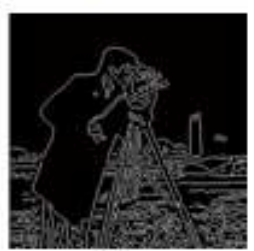

(c)

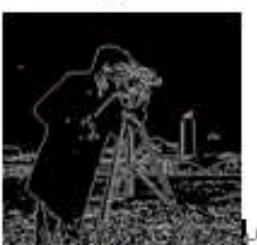

(e).

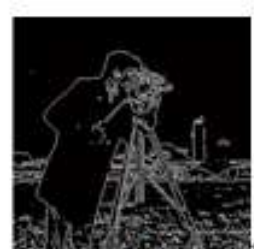

(b)

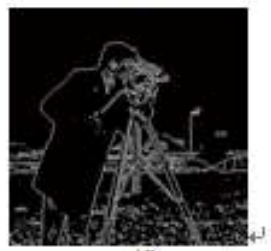

(d)
Figure 6. Edge detection results comparison. (a) The original image. (b) Sobel detection. (c) Canny detection. (d) Scale multiplication in literature[7]. (e) The proposed method

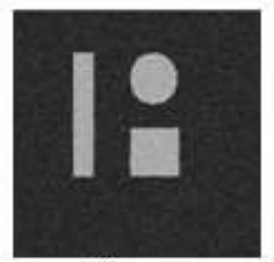

(a)

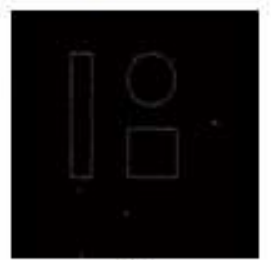

(c)

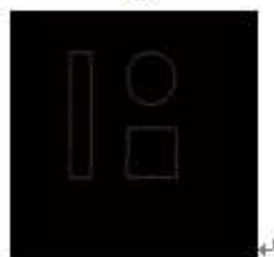

$(e)$.

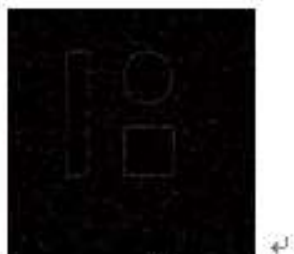

(b)

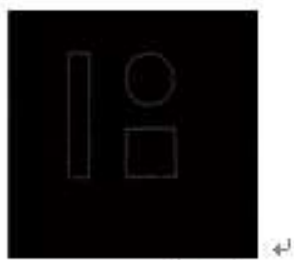

(d)

\section{ACKNOWLEDGMENT}

This work was partly supported by the Chen-Guang Project of Wuhan City (2013072304010826) and the Hubei Natural Science Foundation of China (2013CFB298)

\section{REFERENCES}

[1] J. Canny, "A Computational Approach to Edge Detection," IEEE Trans.Pattern Analysis and Machine Intelligence, vol. 8, pp. 679698, 1986.

[2] P. Melin , O. Mendoza and O. Castillo "An improved method for edge detection based on interval type-2 fuzzy logic", Expert Syst. Appl., vol. 37, no. 12, pp.8527 -8535, 2010.

[3] G. Kim, E. Xing, L. Fei-Fei, and T. Kanade. "Distributed cosegmentation via submodular optimization on anisotropic diffusion", IEEE International Conference on Computer Vision (ICCV), pp. 169-176, Barcelona, Nov. 2011.

[4] O. P.Verma, M.Hanmandlu, K. S. Ashish and P. S. Anil, "A novel fuzzy system for edge detection in noisy image using bacterial foraging", Multidimensional Systems and Signal Processing, vol.24, no.1, pp.181-198, 2013.

[5] I. Sobel, G. Feldman, A 33 isotropic gradient operator for image processing, Presented at a talk at the Stanford Artificial Intelligence Project, 1968.

[6] H. Kong. and S.E. Sarma and F. Tang, "Generalizing Laplacian of Gaussian Filters for Vanishing-Point Detection" Intelligent Transportation Systems,vol.14, no.1, pp.408-418, March 2013.

[7] Paul Bao, Lei Zhang, and Xiaolin Wu. "Canny Edge Detection Enhancement by Scale Multiplication,” IEEE TRANS ON PAMI, vol. 27, no. 9, pp.1485-1490, 2005.

[8] Lijima T. Basic theory on normalization of a pattern (in case of typical one-dimensional pattern). Bulletin of Electrical Laboratory, 1962.

[9] Lindeberg.T, "Scale-space theory: A basic tool for analyzing structures at different scales", Journal of applied statistics, vol.21, no.2, pp. 224-270, 1994

[10] Malik, J., Belongie, S., Leung, T., and Shi, J. "Contour and texture analysis for image segmentation", International Journal of Computer Vision, vol.43,no.1,pp.7-27, 2001.

[11] C.Lopez-Molina, B.De Baets, H.Bustince,J.Sanz and B E.arrenechea, "Multiscale edge detection based on Gaussian smoothing and edge tracking", Knowledge-Based Syst., vol.44, pp.101-111, 2013.

[12] L. Ma, C. Wang and B. Xiao, "Text detection in natural images based on multi-scale edge detection and classification," In the Int. Congress on Image and Signal Processing (CISP), vol.4, pp $1961-1965,2010$.
Figure 7. Edge detection results comparison on noisy image. (a) The original image. (b) Sobel detection. (c) Canny detection. (d) Scale multiplication in literature [7]. (e) The proposed method. 